\title{
Reply: Endocrine therapy in prostate cancer: time for reappraisal of risks, benefits and cost-effectiveness?
}

\author{
D J Rosario*,1 and L Bourke*,2 \\ ${ }^{1}$ Academic Urology Unit, K Floor, Department of Oncology, Royal Hallamshire Hospital, Glossop Road, University of Sheffield, \\ Sheffield S11 7FE, UK and '2Department of Primary Care and Public Health, Barts and The London School of Medicine and \\ Dentistry, Queen Mary University of London, London E1 2AT, UK
}

Sir,

We are very grateful to Shah et al, (2013) for their interest in our review of ADT for prostate cancer. The aim of the review was to highlight the spiralling costs of ADT and the likely increase in adverse cardiovascular effects from the most commonly used forms of ADT (LHrH analogues and orchidectomy) and in particular to draw attention to the lack of research investigating which men are at most risk and how to reduce such risk.

In this context, the PATCH study (Langley et al, 2013) is likely to be a valuable addition to the literature, but may also represent a missed opportunity. The 'cardiovascular exclusion criteria' for this study are extensive. Thus, the results are not going to be generalisable to all men with advanced prostate cancer being considered for ADT, given the age range of the men affected and the recognised association between prostate cancer mortality and risk factors for cardiovascular disease, particularly obesity. The initial study reported in this low-risk cohort was not powered to detect a difference in cardiovascular events between the two groups (LHrHa and oestrogen patches). However, Langley et al (2013) report the incidence of cardiovascular events at a median follow-up of 19 months (IQR 12-31, minimum 3 months) in the LHRHa group as $7.1 \%$ (95\% CI 2.7-14.9) and in the oestrogen-patches group as $10.1 \%$ (95\% CI $6.0-15.6)$. The number of events in both groups seems rather high, with the rate of cardiovascular events being $2.9 \%$ higher in the oestrogen-patch group than in the LHRHa group (95\% CI -4.2 to 10.1 ).
Whereas treatment with parenteral oestrogen may represent an advance for certain adverse effects of ADT as well as possibly being more cost-effective, evidence is still lacking. We stand by the conclusion of our review, namely that a better understanding of the risks and any excess treatment cost associated with ADT is imperative to inform on the cost-effectiveness of various methods of achieving castration and whether interventions are available to reduce their risks. We hope the PATCH study will go some way towards providing further evidence on cost-effectiveness, but are disappointed it does not address cardiovascular risk in a generalisable population.

\section{REFERENCE}

Langley RE, Cafferty FH, Alhasso AA, Rosen SD, Sundaram SK, Freeman SC, Pollock P, Jinks RC, Godsland IF, Kockelbergh R, Clarke NW, Kynaston HG, Parmar MKB, Abel PD (2013) Cardiovascular outcomes in patients with locally advanced and metastatic prostate cancer treated with luteinising-hormonereleasing-hormone agonists or transdermal oestrogen: the randomised, phase 2 MRC PATCH trial (PR09). Lancet Oncol 14(4): 306-316.

Shah SIA, Abel PD, Langley RE, Cafferty FH (2013) Comment on 'Endocrine therapy in prostate cancer: time for re-appraisal of risks, benefits and cost-effectiveness? Br J Cancer 108(10): 2192-2193. 